\title{
Erros do modelo WRF associados às parametrizações de turbulência em noites fracamente estáveis
}

\author{
WRF model errors associated with the turbulence parameterizations in weakly \\ stable nights
}

\author{
Adriano Battisti ${ }^{1}$, Felipe Denardin Costa ${ }^{2}$, Otávio Costa Acevedo ${ }^{3}$, Viviane da Silva Guerra ${ }^{4}$, \\ Thaís Freitas Dill ${ }^{1}$
}

\author{
${ }^{1}$ Pós-Graduação em Meteorologia, Universidade Federal de Santa Maria, Santa Maria, RS, Brasil \\ ${ }^{2}$ Professor da Pós-Graduação em Engenharia, Campus Alegrete, Universidade Federal do Pampa, Alegrete, RS, Brasil \\ ${ }^{3}$ Professor do Curso de Meteorologia, Universidade Federal de Santa Maria, RS, Brasil \\ ${ }^{4}$ Pós-Graduação em Física, Universidade Federal de Santa Maria, Santa Maria, RS, Brasil
}

\begin{abstract}
Resumo
A qualidade da previsão de temperaturas noturnas por diferentes parametrizações de camada limite atmosférica é avaliada. Para isso, foi utilizado o modelo WRF, sendo que se considerou três representações diferentes para a turbulência no modelo: Bougeault-Lacarrère (BOU), Mellor-Yamada-Janjic (MYJ) e Yonsei University (YSU). Para YSU e BOU se avaliou a previsão com um espaçamento de grade de $12 \mathrm{~km}$ e para MYJ se avaliou a grade de $12 \mathrm{e} 4 \mathrm{~km}$, centradas no estado do Rio Grande do Sul, no sul do Brasil. Foram utilizadas as noites mais turbulentas, menos estáveis, a fim de investigar quais os problemas associados ao erro de previsão neste ambiente. O parâmetro número de Richardson foi responsável por classificar as noites na sua estabilidade. Os erros são confrontados com o erro da previsão de vento e com os parâmetros de estabilidade. A comparação mostra que o modelo subestima consistentemente as temperaturas noturnas nessas noites e o aumento da resolução não resultou em uma melhora significativa da previsão. Além disso, o erro do vento para as situações de menor estabilidade é pequeno, o que indica que há problema na solução da turbulência pelos esquemas de camada limite atmosférica neste tipo de ambiente.
\end{abstract}

Palavras-chave: temperaturas noturnas, parametrização de turbulência, regime fracamente estável, modelo WRF, espaçamento de grade.

\begin{abstract}
The quality of nocturnal temperatures forecast by different parameterizations of the atmospheric boundary layer is evaluated. To do that, the WRF model is used. Three different turbulence representation have been considered: Bougeault-Lacarrère (BOULAC), Mellor-Yamada-Janjic (MYJ) and Yonsei University (YSU). For YSU and BOULAC the forecast have been evaluated with a grid spacing of $12 \mathrm{~km}$ and for MYJ a grid spacing with $12 \mathrm{~km}$ and one with $4 \mathrm{~km}$, centered on state of Rio Grande do Sul, in south Brazil. The more turbulent nights have been used, the less stables, to investigate what is the problems associated to the forecast error in this environment. The Richardson number parameter was the responsible by class the nights according to its stability. The errors are compared with the quality of wind speed forecast and stability parameters. The comparison shows which the model consistently underestimate nocturnal temperatures in these nights and the resolution upgrade did not work in a significative forecast improvement. Furthermore, the windy speed error in the situations with less stability is small. That indicates a existence of a problem associated with the turbulence solution by the atmospheric boundary layer schemes in this type of environment.
\end{abstract}

Keywords: nocturnal temperatures, turbulence parameterization, weakly stable regime, WRF model, grid spacing. 


\section{Introdução}

A temperatura, como uma das principais variáveis meteorológicas, é importante para diversas atividades humanas, inclusive no período noturno. Como fenômenos de maior impacto que costumam ocorrer na camada limite estável ou noturna e que estão associados a esta variável, temos as geadas, que afetam a agricultura e pecuária, e nevoeiros, que afetam os transportes. Além disso, as temperaturas noturnas próximas à superfície estão diretamente associadas à parametrização de turbulência nos modelos de previsão numérica. Entretanto, nas atuais parametrizações de camada limite atmosférica dos modelos de previsão, a representação dos processos de camada limite estável é ainda de baixa qualidade e os resultados obtidos para melhorar os esquemas são poucos (Steeneveld et al., 2006). Tanto no regime de forte como no de fraca turbulência, que estão diretamente associados à velocidade do vento, as características dos fluxos noturnos não são simples e nem lineares (Sun et al., 2012).

Portanto, o objetivo deste trabalho é investigar a qualidade da previsão de temperatura mínima em modelos de mesoescala. Para isso, além de diferentes formulações de camada limite, são comparadas duas resoluções diferentes por meio de integrações utilizando o modelo Weather Research and Forecasting (WRF). São investigadas as noites com vento mais forte, ou seja, noites características do regime fracamente estável.

\section{Materiais e Métodos}

Neste trabalho foram realizadas integrações com o modelo WRF, do mesmo modo que são feitas em uma previsão operacional. Para isso, utilizou-se como condições iniciais e de contorno a análise e as previsões de 6 em 6 horas do modelo global Global Forecast System (GFS) para os dias estudados. Três domínios são utilizados, centrados no estado do Rio Grande do Sul (Figura 1), com espaçamento de grade: 48, 12 e $4 \mathrm{~km}$. Os menores, de maior resolução, estão aninhados nos maiores, com menos resolução. A projeção usada é a de Lambert. A resolução do relevo fornecida para interpolação para os dois domínios maiores é 2 minutos, enquanto para o menor 30 segundos. As simulações foram realizadas para todos as noites do mês de Julho de 2012, com início às 00 UTC (Tempo Universal Coordenado, do inglês Universal Time Coordinated) e término às 12 UTC de cada dia, ou seja, das 21 às 09 hora local.

São comparadas três parametrizações: BougeaultLacarrère (BOU); Mellor-Yamada-Janjic (MYJ); e YonseiUniversity (YSU). Para BOU e YSU se retira as variáveis da grade de $12 \mathrm{~km}$; para MYJ se utiliza as variáveis da grade de 12 e de $4 \mathrm{~km}$, o que configura quatro diferentes

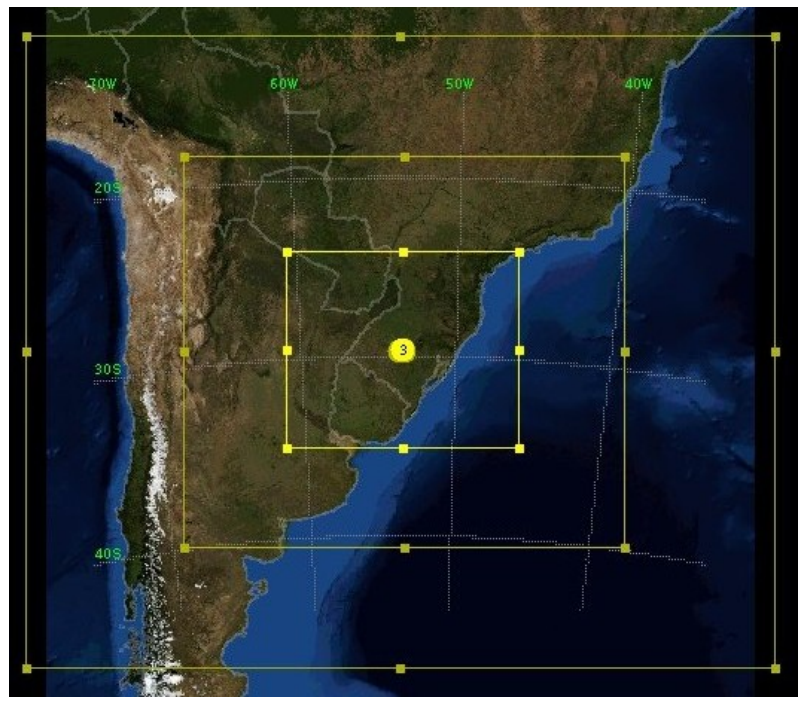

Figura 1: Domínio das 3 grades usadas nas integrações.

modos de integração, totalizando 120 simulações. Os valores horários de temperatura e vento próximo à superfície das 00 às 06 hora local são comparados com as observações de 26 estações automáticas de superfície do Instituto Nacional de Meteorologia (INMET). A localização das estações pode ser vista na figura (2). Além disso, os erros são confrontados com parâmetros de estabilidade.

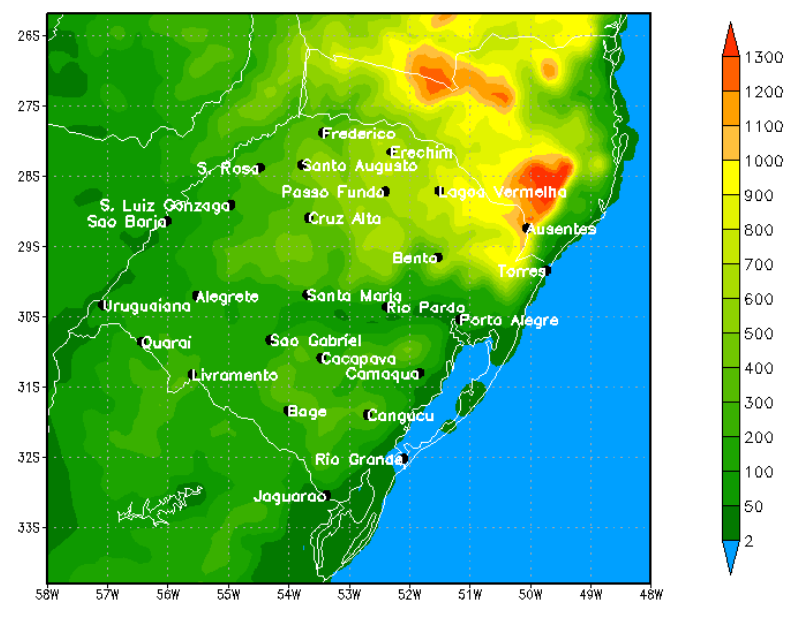

Figura 2: Localização das estações e topografia da região em estudo (m).

Como ferramenta estatística se utiliza o erro médio, que é simplesmente a média dos dados modelados menos observados, e a raiz do erro quadrático médio (REQM), dada por:

$$
R E Q M=\sqrt{\frac{1}{N} \sum_{k=1}^{N}(\operatorname{sim}-o b s)^{2}}
$$




\section{Resultados e Discussão}

Para se caracterizar as noites de acordo com a estabilidade de cada uma delas se utilizou um número de Richardson estadual, $R i_{\text {estadual }}$, calculado com os dados presentes nas 26 estações de superfície:

$$
R i_{\text {estadual }}=\frac{\theta_{\max }-\theta_{\min }}{\overline{V_{o b s}}}
$$

Em que $\theta_{\max }$ é a temperatura potencial máxima entre as 26 estações e entre os 7 horários, 00 às 06 local; $\theta_{\min }$ é a temperatura potencial mínima da noite; e $\overline{V_{o b s}}$ é a velocidade média do vento à $10 \mathrm{~m}$, médio da noite; $R i_{\text {estadual }}$ é calculado para cada noite. Como este número de Richardson não é local e cada valor dele é calculado com os dados de todas as estações e de todos os horários de cada noite, ele não apresenta os valores clássicos que costumam ocorrer localmente. Entretanto, ele caracteriza eficientemente a estabilidade característica de cada noite no estado do Rio Grande do Sul, o que permite classificar quais foram as noites menos estáveis. Se utiliza, portanto, as noites com $R i_{\text {estadual }}$ menor que 5 como sendo as noites menos estáveis, o que dá um número de 13 noites para se fazer o estudo entre as 31 simuladas.

Ao se comparar a temperatura observada e prevista entre as noites aqui classificadas como fracamente estáveis, se percebe que na maioria dos casos, para as quatro situações estudadas, o modelo subestima consistentemente a temperatura no período noturno, a partir do erro médio da noite apresentado na tabela 1 . Além disso, podemos ver pela REQM que o menor erro para as noites menos estáveis foi a integração com a parametrização BOULAC, uma parametrização mais turbulenta (Battisti, 2014). Além disso, o aumento da resolução do modelo, que exigiu muito mais custo computacional, pouco melhorou a previsão de temperaturas noturnas.

Para relacionar o erro da previsão com um possível erro do vento em função da estabilidade do ambiente, foi calculado um número de Richardson regional, $R i_{\text {regional }}$, com um valor para todos os horários analisados, o que foi possível ao se fazer da seguinte forma:

$$
R i_{\text {regional }}=\frac{\theta_{\text {max }}-\theta_{o b s}}{V_{\text {obs }}}
$$

Em que $\theta_{\text {max }}$ é a temperatura potencial máxima entre as 26 estações; $\theta_{o b s}$ é a temperatura potencial de cada estação; e $V_{o b s}$ é a velocidade do vento à $10 \mathrm{~m}$ de cada estação; isso para todos os dados, ou seja, para cada horário de cada noite e cada estação. Este $R i_{\text {regional }}$ é usado com o propósito de, mesmo que aproximadamente, quantificar a estabilidade atmosférica local a partir dos dados observados.

Pela figura 3, que confirma a subestimação de temperatura por parte do modelo, se percebe que o erro aumenta quanto menos estável for o ambiente. Sendo
Tabela 1: Erro médio para cada noite e cada modo de integração, além da REQM de cada modo.

\begin{tabular}{lcccc}
\hline noite & BOULAC & MYJ & YSU & MYJ 4 km \\
\hline 1 & -1.49 & -1.85 & -1.47 & -1.73 \\
2 & -2.19 & -2.85 & -2.46 & -2.86 \\
3 & -0.26 & -0.54 & -0.12 & -0.56 \\
5 & -0.43 & -1.24 & -0.99 & -1.22 \\
6 & 0.35 & -0.51 & 0.08 & -0.59 \\
9 & -1.89 & -1.89 & -1.59 & -1.64 \\
10 & -1.33 & -1.72 & -1.39 & -1.82 \\
13 & -0.81 & -1.30 & -1.14 & -0.91 \\
15 & -0.09 & -0.40 & -0.19 & -0.73 \\
16 & -0.44 & -1.11 & -0.65 & -0.91 \\
21 & -1.64 & -1.83 & -1.34 & -1.75 \\
30 & 0.03 & -0.71 & -0.64 & -0.61 \\
31 & 0.45 & -1.24 & -0.83 & -1.35 \\
\hline média do erro & -0.75 & -1.32 & -0.98 & -1.28 \\
REQM & 2.44 & 2.57 & 2.46 & 2.55 \\
\hline
\end{tabular}

que para o período noturno, ambientes menos estáveis costumam ter vento mais forte. Portanto, pensou-se que a velocidade do vento para estes casos poderia estar sendo subestimada. Mas não é o que ocorre. Na figura 4 vemos que o erro do vento, que é superestimado, não é muito grande para ambiente menos estável. E isto para todos os esquemas.

Shin e Hong (2011), ao analisarem a performance de cinco esquemas de parametrização no modelo WRF, também mostram que não houve algum que simulasse a turbulência noturna satisfatoriamente, dando mais evidência que existem processos na camada limite estável ainda não bem representados.

\section{Conclusões}

A qualidade da previsão de temperaturas noturnas por 3 esquemas de parametrização no modelo WRF foi analisada, sendo uma delas com duas resoluções de integração. O estudo se caracterizou por analisar as noites menos estáveis, dentre um mês inteiro, e por uma comparação que englobou estações de todas as regiões do estado do Rio Grande do Sul.

Analisando-se o erro da previsão, evidenciou-se que o modelo subestima consistentemente as temperaturas noturnas nas noites menos estáveis. Além disso, a parametrização que obteve o maior erro entre as integrações de $12 \mathrm{~km}$ de espaçamento de grade foi MYJ, sendo que integrações com espaçamento de $4 \mathrm{~km}$ não foram suficientes para deixá-la com menor erro que BOULAC e YSU; esta última apresentando o menor erro. Portanto, o custo computacional exigido, de primeiro momento, parece não compensar na melhora da previsão. 


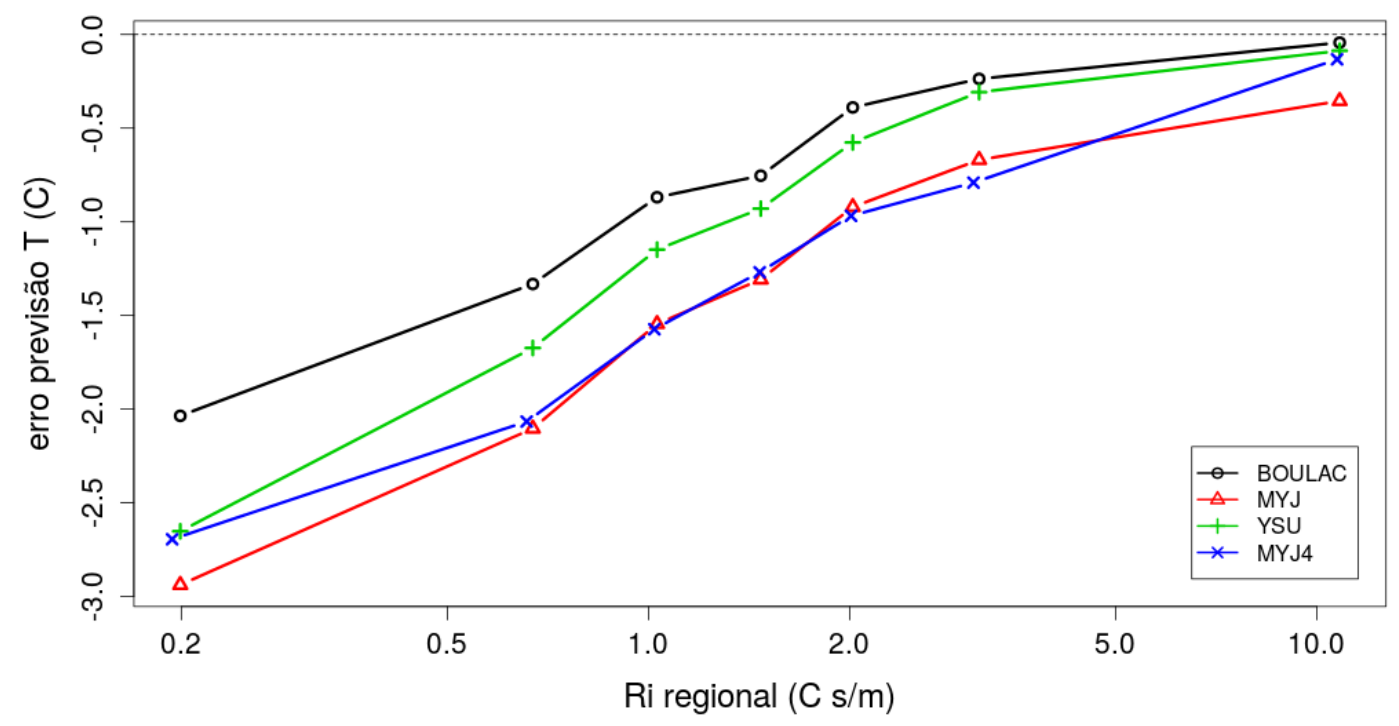

Figura 3: Erro médio em blocos de previsão de temperatura (T) rm função de $R i_{\text {regional }}$. Cada ponto representa 300 dados, observados e previstos.

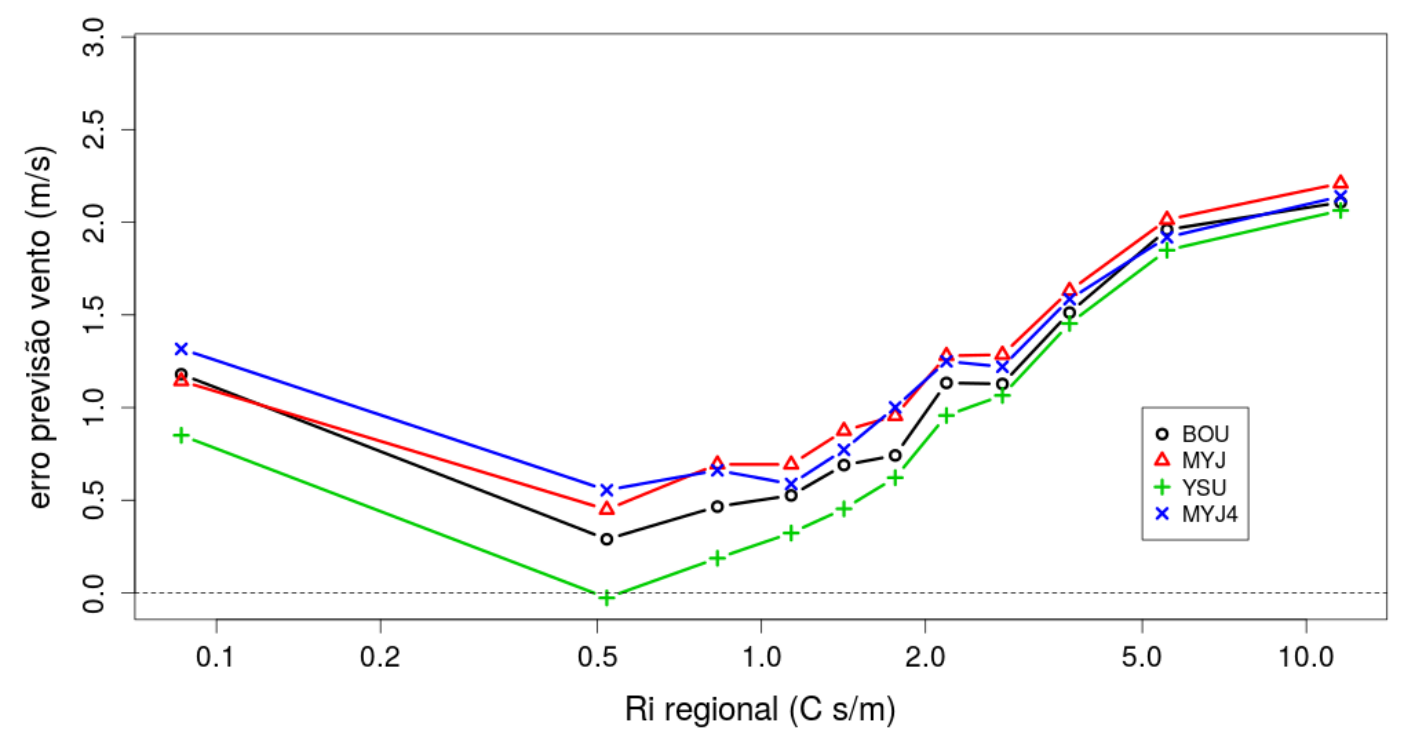

Figura 4: O mesmo que na figura 3, mas para o erro da velocidade do vento.

Estes grandes erros nas noites menos estáveis, que costumam apresentar vento mais forte, levaram a pensar que o modelo poderia estar simplesmente errando a velocidade do vento. Entretanto, observou-se que justamente nos ambientes menos estáveis, o erro do vento é pequeno, enquanto o erro de temperatura cresce. $\mathrm{Ou}$ seja, o modelo não está conseguindo reproduzir em tur- bulência o momento que lhe é fornecido. Portanto, isto parece ser um problema dos próprios esquemas de camada limite atmosférica, que estão deixando o ambiente muito frio. Isto poderia estar acontecendo devido a processos como turbulência intermitente, estudada em Costa et al. (2011), e processos não turbulentos como submeso, que é descrita em Mahrt (2009), que podem 
não estar sendo reproduzidos pelos esquemas.

\section{Referências}

Battisti, A. (2014). Parametrização de Turbulência na Previsibilidade de Temperaturas Mínimas em um Modelo de Mesoescala. Mestrado em meteorologia, Universidade Federal de Santa Maria, Santa Maria.

Costa, F. D., Acevedo, O. C., Mombach, J. C. M., Degrazia, G. A. (2011). A simplified model for intermittent turbulence in the nocturnal boundary layer. Journal of the Atmospheric Sciences, 68(8), 1714-1729.

Mahrt, L. (2009). Characteristics of submeso winds in the stable boundary layer. Boundary-Layer Meteorology, 130(1), 1-14.

Shin, H. H., Hong, S. Y. (2011). Intercomparison of planetary boundary-layer parametrizations in the WRF model for a single day from CASES-99. BoundaryLayer Meteorology, 139(2), 261-281.

Steeneveld, G. J., van de Wiel, B. J. H., Holtslag, A. A. M. (2006). Modeling the evolution of the atmospheric boundary layer coupled to the land surface for three contrasting nights in CASES-99. Journal of the Atmospheric Sciences, 63(3), 920-935.

Sun, J., Mahrt, L., Banta, R. M., Pichugina, Y. L. (2012). Turbulence regimes and turbulence intermittency in the stable boundary layer during CASES-99. Journal of the Atmospheric Sciences, 69(1), 338-351. 